\title{
Survivor, family and professional experiences of psychosocial interventions for sexual abuse and violence: a qualitative evidence synthesis (Protocol)
}

Brown SJ, Khasteganan N, Carter GJ, Brown K, Caswell RJ, Howarth E, Feder G, O'Doherty L

Brown SJ, Khasteganan N, Carter GJ, Brown K, Caswell RJ, Howarth E, Feder G, O'Doherty L.

Survivor, family and professional experiences of psychosocial interventions for sexual abuse and violence: a qualitative evidence synthesis (Protocol).

Cochrane Database of Systematic Reviews 2020, Issue 6. Art. No.: CD013648.

DOI: 10.1002/14651858.CD013648. 
TABLE OF CONTENTS

HEADER 1

ABSTRACT

BACKGROUND

OBJECTIVES

METHODS

ACKNOWLEDGEMENTS

REFERENCES

APPENDICES

HISTORY

CONTRIBUTIONS OF AUTHORS

DECLARATIONS OF INTEREST

SOURCES OF SUPPORT 
[Qualitative Protocol]

\section{Survivor, family and professional experiences of psychosocial interventions for sexual abuse and violence: a qualitative evidence synthesis}

Sarah J Brown 1,2, Nazanin Khasteganan ${ }^{1}$, Grace J Carter ${ }^{1}$, Katherine Brown ${ }^{3}$, Rachel J Caswell ${ }^{4}$, Emma Howarth ${ }^{5}$, Gene Feder ${ }^{6}$, Lorna O'Doherty 1,7

${ }^{1}$ Faculty of Health and Life Sciences, Coventry University, Coventry, UK. ${ }^{2}$ Faculty of Arts, Business and Law, Law School, USC: University of the Sunshine Coast, Sippy Downs, Australia. ${ }^{3}$ Department of Psychology and Sports Science, University of Hertfordshire, Hatfield, UK. ${ }^{4}$ Sexual Health and HIV Medicine, University Hospitals Birmingham NHS Foundation Trust, Birmingham, UK. ${ }^{5}$ School of Psychology, University of East London, London, UK. ${ }^{6}$ Centre for Academic Primary Care, Population Health Sciences, Bristol Medical School, University of Bristol, Bristol, UK. ${ }^{7}$ Department of General Practice, The University of Melbourne, Melbourne, Australia

Contact address: Sarah J Brown, Sarah.Brown@Coventry.ac.uk,sbrown2@usc.edu.au.

Editorial group: Cochrane Developmental, Psychosocial and Learning Problems Group.

Publication status and date: New, published in Issue 6, 2020.

Citation: Brown SJ, Khasteganan N, Carter GJ, Brown K, Caswell RJ, Howarth E, Feder G, O'Doherty L. Survivor, family and professional experiences of psychosocial interventions for sexual abuse and violence: a qualitative evidence synthesis (Protocol). Cochrane Database of Systematic Reviews 2020, Issue 6. Art. No.: CD013648. DOI: 10.1002/14651858.CD013648.

Copyright $\odot 2020$ The Cochrane Collaboration. Published by John Wiley \& Sons, Ltd.

\section{A B S T R A C T}

\section{Objectives}

This is a protocol for a Cochrane Review (qualitative). The objectives are as follows:

This review will gather and synthesise the experiences of interventions for survivors of sexual abuse and violence, their families, as well as the professionals who deliver them.

Specifically, this review seeks to:

1. identify, appraise and synthesise qualitative studies exploring the experiences of child and adult survivors of sexual abuse and violence, and their caregivers, regarding psychosocial interventions aimed at supporting survivors and preventing negative health outcomes in terms of benefits, risks/harms and barriers;

2. identify, appraise and synthesise qualitative studies exploring the experiences of professionals who deliver psychosocial interventions for sexual abuse and violence in terms of perceived benefits, risks/harms and barriers for survivors and their families/caregivers;

3. develop a conceptual understanding of how different factors influence uptake, dropout or completion, and outcomes from psychosocial interventions for sexual abuse and violence;

4. develop understanding of how features and types of interventions respond to the needs of different user/survivor groups (e.g. age groups; types of abuse exposure; migrant populations) and contexts (healthcare/therapeutic settings; low- and middle-income countries (LMICs)); and

5. explore how the findings of this review can enhance our understanding of the findings from the linked and related reviews assessing the effectiveness of interventions aimed at supporting survivors and preventing negative health outcomes. 


\section{B A C K G R O U N D}

\section{Description of the issue}

Sexual violence is defined as "any sexual act, attempt to obtain a sexual act, unwanted sexual comments or advances, or acts to traffic, or otherwise directed, against a person's sexuality using coercion, by any person regardless of their relationship to the victim, in any setting, including but not limited to home and work." (Jewkes 2002). Coercion includes a wide range of behaviours including physical force, psychological intimidation, threats, and blackmail. Coercion also occurs when an individual is unable to consent; for example, because they are too young, or unable to understand the situation, or incapacitated due to drugs or alcohol, or are asleep (Jewkes 2002). Sexual violence includes a wide range of acts including rape, defined as "physically forced or otherwise coerced penetration - even if slight - of the vulva or anus, using a penis, other body parts or an object" (Jewkes 2002), attempted rape and "other forms of assault involving a sexual organ, including coerced contact between the mouth and penis, vulva or anus." (Jewkes 2002). When children are victims of sexual violence, this is typically referred to as child sexual abuse (CSA). For this reason, and given that many adult victims of sexual violence do not perceive their victimisation as 'violence', we will use 'sexual abuse and violence' throughout this qualitative evidence synthesis (QES).

Syndemic frameworks (with concurrent or sequential diseases that additively increase negative health consequences) theorise about the ways in which experiences of abuse and violence and other phenomena related to health, cultural, social and economic factors may co-occur and exacerbate each other (Brennan 2012; Singer 2003). Structural factors, like lack of housing, poverty and immigration status; and social aspects, such as gender identities, sexual identities, ethnicity, disability, history of exploitation or sex work and poor support systems; can interact with experiences of abuse to produce health inequities and reinforce the burden of disease (Willen 2017). Research evidence shows that victims of CSA are at increased risk of experiencing multiple forms of child maltreatment and abuse, and that such polyvictimisation is a key determinant in the development of negative health and behaviour outcomes (e.g. Ford 2010; Leach 2016; Turner 2016). These factors also mean that the experiences of those affected by constellations of social, political, health and economic factors are less likely to be represented in research and prevalence studies. Sexual abuse and violence is a significantly under-reported problem in all populations, but these issues mean that it is particularly under-studied and reported in vulnerable and under-represented populations and during times of conflict and war; hence, it is difficult to fully understand the extent of the problem. For example, among 2013/14 Crime Survey for England and Wales respondents, only $17 \%$ of the sexual assaults experienced since the age of 16 years were reported to the police (ONS 2015). Similarly, just $23 \%$ of the 323,450 rapes or sexual assaults against individuals aged 12 years or older disclosed in the US National Crime Victimization Survey in 2016 had been reported to the police (Morgan 2017). Following a review of research studies, London and colleagues showed that most adults (55\% to $69 \%$ ) who identified as survivors of CSA did not disclose this abuse to anyone during childhood, with only $5 \%$ to $13 \%$ reporting the abuse to the authorities (London 2008). In fact, many (10\% to $46 \%$ ) reported that the disclosure of the abuse for the research study was their first disclosure.
Estimates of prevalence vary widely depending on the definitions used, method of data collection, and populations targeted. For example, there are more population-based survey data available to estimate sexual abuse and violence perpetrated by intimate partners, compared to that perpetrated by non-partners (WHO/ PAHO 2012). The lifetime prevalence of sexual violence perpetrated by an intimate partner reported by women aged 15 to 49 years in the WHO multi-country study ranged from $6 \%$ in Japan to $59 \%$ in Ethiopia (WHO 2005). In the same study, $0.3 \%$ to $12 \%$ of women reported having been forced, after the age of 15 years, to have sexual intercourse or to perform a sexual act by someone other than an intimate partner (WHO 2005). Social and legal marginalisation, exacerbated by gender-defined services, stigma, discrimination and studies with small sample sizes and varying definitions mean that the experiences of sexual abuse and violence by trans gender people (Wirtz 2018) and men are hidden and poorly understood. In relation to non-heterosexual populations, the 2010 US National Intimate Partner and Sexual Violence Survey indicated that people who identify as nonheterosexual are disproportionately victimised, with one in five bisexual women reporting rape by a partner (compared to one in 10 heterosexual women) (Waters 2013). Higher rates of sexual violence are also experienced by gay men and bisexual men compared to heterosexual men (Waters 2013). Estimates of sexual abuse and violence prevalence using reports of perpetrators are rare. A crosssectional survey of a randomly selected sample of men in South Africa revealed that $14 \%$ had raped their current or former wife or girlfriend, while one in five reported raping a woman who was not a partner (i.e. a stranger, acquaintance or family member) (Jewkes 2011). A meta-analysis of 65 studies covering 22 countries showed that $7 \%$ of men and $19 \%$ of women had suffered sexual abuse prior to 18 years of age (Pereda 2009).

Sexual abuse and violence has devastating effects on adult and child victims, their families and communities. In the US National Epidemiologic Survey on Alcohol and Related Conditions ( $\mathrm{n}=$ 34,653; Pietrzak 2011), sexual assault was ranked among the top three most traumatic life events. Extensive immediate and longterm consequences for adult and child victims include injuries, substance misuse, eating disorders, post-traumatic stress disorder (PTSD), anxiety, depression, self-harm and suicidality (WHO 2013). Sexual and reproductive health problems for women include unwanted pregnancy and sexually transmitted infections (WHO 2013), while physical health consequences for men include genital and rectal injuries and erectile dysfunction (Tewkesbury 2007). The mental health burden is substantial and similar across male and female victims (Guina 2019; Tewkesbury 2007; WHO 2013). PTSD, a psychiatric disorder that can follow exposure to psychological trauma, is associated with intrusive memories, nightmares, avoidance, and problems with sleep and concentration (Lerman 2019). Individuals with PTSD were four times more likely to report exposure to sexual assault than those not affected by PTSD, and $13 \%$ of women with PTSD had lifetime experience of sexual assault (Pietrzak 2011). No differences in PTSD symptoms and severity have been found between men and women who have experienced sexual trauma (Guina 2019). Other mental health consequences include alcohol use disorders, eating disorders, anxiety, depression, self-harm and suicidality (WHO 2013).

Sexual abuse and violence also has considerable social and economic costs affecting individuals' capacities to participate in family, community and economic life (e.g. to engage in work). 
in addition to the health and mental health burden, there are lost productivity, police, criminal justice, social and other service costs. Each adult rape in the UK has been estimated to cost over GBP 73,000 from psychological damage to a person, the physical impacts of associated injuries and illnesses, health service use, and economic losses (Dubourg 2005). The US Centers for Disease Control and Prevention (CDC) estimated that the lifetime cost of rape in the USA was USD 122,461 per victim, which amounted to a population economic burden of almost USD 3.1 trillion (Peterson 2017). Estimates suggest that in the UK, CSA exposure leads to GBP 182 million in health spending annually (Saied-Tessier 2014), and in the USA the lifetime economic burden is approximately USD 9.3 billion (Letourneau 2018). Additional impacts include impacts on families, capacities to parent and intergenerational transmission of trauma and violence. Hence, providing accessible, evidence-based interventions for victims is essential to limit the consequences of sexual abuse and violence. Sexual abuse and violence silences and disempowers victims, and providing opportunities for individuals to talk about their trauma and experiences of the services and interventions that they have received provides an important opportunity for them to speak out and help inform the development, improvement and increased accessibility of services and interventions. Hence, it is important to understand how survivors, their families, and professionals view psychosocial interventions, in order to supplement evaluation reviews and to understand the benefits and harms of interventions, as well as their appropriateness and acceptability, from the stakeholders' perspectives.

\section{Description of the intervention}

It is well established that experiencing sexual abuse and violence can have a range of detrimental impacts for those who have experienced it directly, and beyond the individual directly affected. It impacts families and individuals supporting survivors. The nature of the support available for survivors is linked to how we understand and conceptualise the harm experienced through exposure to sexual abuse and violence. There are a wide range of interventions that support and respond to those who have experienced sexual abuse and violence.

In the early 1970s, interventions were developed for individuals who had experienced sexual abuse and violence. These early interventions arose from a crisis theory orientation (e.g. Burgess 1974). Such interventions were pivotal to informing the development of advocacy organisations (Koss 1987). However, there has been limited evidence to demonstrate how effective these interventions were, with some research studies suggesting that more intensive treatment was needed in order to address effectively the chronic symptoms experienced by some survivors (Kilpatrick 1983). Throughout the 1970s, evidence-based anxiety treatments were developed for survivors of sexual abuse and violence, including cognitive-behavioural interventions such as stress inoculation training (SIT; Veronen 1983), prolonged exposure therapy (PET; Foa 1986) and cognitive processing therapy (CPT; Resnick 1997; see Vickerman 2009 for a review of these). Situated within a trauma-response theoretical model (Goodman 1993; Herman 1992), behavioural therapies, including eye movement desensitisation reprocessing (EMDR; Rothbaum 1997; Shapiro 1995) were introduced and evaluated.

The interdependent nature of responses given by individuals and community organisations can lead to individuals each having different types of experiences, which are dependent on their ecological circumstances. This has led to scholars of violence against women and girls emphasising the importance of adopting an ecologically-informed trauma model of rape recovery (Koss 1991; Neville 1999) that acknowledges the different systems within which social and psychological responses are given to support this population. This has been accounted for in Kelly's ecological theory (Kelly 1966; Kelly 1968; Kelly 1971). Harvey 1996 and Koss 1991 adapted Kelly's ideas to develop their own ecological model of rape recovery, which was used by Campbell and colleagues in their evaluation of how legal, medical, and mental health systems respond to the needs of survivors and what factors can impact their psychological, physical and sexual health outcomes (Campbell 1998; Campbell 1999; Campbell 2001; Campbell 2004). Similarly, the World Health Organization (WHO) (Jewkes 2002; Krug 2002) and CDC (CDC 2004) adapted this approach to address the prevention of gender-based violence. Thus, there are a wide variety of interventions that have been developed to support or respond to (or both) individuals who have experienced sexual abuse and violence. These include supportive therapies, whereby counsellors, and/or specific sexual assault/rape support workers, advocates or advisors provide this population with information, advice and support.

Psychosocial interventions are defined as "interpersonal or informational activities, techniques, or strategies that target biological, behavioral, cognitive, emotional, interpersonal, social, or environmental factors with the aim of improving health functioning and well-being" (IOM 2015). Such interventions vary considerably as they target different combinations of the aforementioned factors. For example, group education sessions (e.g. Dognin 2017) and brief video-based interventions that provide psychoeducation and model coping strategies have been developed for survivors undergoing a sexual assault nurse examination (Miller 2015). Furthermore, in the UK, Sexual Assault Referral Centres (e.g. NHS 2015 and Vandenberghe 2018) provide a range of initial response and support services. This includes the involvement of independent sexual violence advisors (ISVAs) who are non-psychologists trained to provide survivors with tailored support to address their needs, accurate and impartial information, and support before, during and after court (Home Office 2017). It is important to note that the discussion of the incident prior to court proceedings can be seen as prejudicial to a trial (CPS 2002) and so is often discouraged. In light of this, psychosocial interventions that are tailored to avoid such discussion can be a vital source of support to rape and sexual assault victims in the pretrial period.

In this QES, we will focus on the qualitative components of studies that explore the experiences of survivors, their families and professionals in relation to psychosocial interventions targeted at individuals who have experienced sexual abuse and violence. This will include a wide range of psychosocial interventions that target recovery from adult or child (or both) sexual abuse and violence. Women have been the primary focus as recipients of interventions and services for sexual abuse and violence survivors, whilst male, transgender and gender nonconforming or nonbinary populations experience significant barriers in accessing such interventions. Unsurprisingly, these differences in gender regarding intervention recipients have been reflected in the intervention evaluation literature, whereby the samples used in intervention evaluation studies are usually female. In comparison, non-female populations have received little attention in evaluation

Survivor, family and professional experiences of psychosocial interventions for sexual abuse and violence: a qualitative evidence 
studies. There is further imbalance in relation to ethnicity, whereby evaluation samples are predominantly White/Caucasian women and girls, whilst many subgroups, including minority ethnic groups and migrant individuals, remain hidden in both practice and research. This review is pertinent to bringing together experiences of interventions across studies among individuals typically underrepresented in research, who share certain social, gender, ethnic and economic characteristics, to examine the acceptability and appropriateness of interventions for subgroups of survivors.

The review will focus on any setting where a person has received an intervention or a professional has delivered an intervention aimed at supporting a survivor or family member in the aftermath of an experience of sexual abuse and violence. For all studies, interventions of any duration or frequency of treatment will be included. For the purposes of this review, we will include a wide range of psychosocial interventions (for definitions, see the list of psychological therapies on the Cochrane Common Mental Disorders (CCMD) website: cmd.cochrane.org/ psychological-therapies-topics-list).

1. Integrative therapies, including SIT, PET, and CPT

2. Behaviour therapies such as EMDR and relaxation techniques

3. Formal cognitive behavioural therapy (CBT), trauma focused CBT (TF-CBT) and CBT-based techniques

4. Third wave CBT (e.g. acceptance and commitment therapy, mindfulness)

5. Humanistic therapies (e.g. supportive and non-directive therapy)

6. Other psychologically-orientated interventions (e.g. art therapy, meditation, trauma-informed body-based practices (e.g. embodied relational therapy, yoga and Tai Chi), narrative therapy)

7. Psychosocial interventions, including those delivered by mentors, support workers, advisors or advocates (such as ISVAs in the UK), support groups, and coping interventions

For all interventions, mode of intervention delivery may include face-to-face, telephone or computer-based delivery. We will include in this review studies reporting individual or group delivery of the intervention.

We will include any studies that include feedback from or perspectives of the stakeholder groups, regardless of whether the study (or broader research project) included a comparison or not.

The proposed review will synthesise qualitative evidence, including information on feasibility and acceptability, experience and outcomes of interventions to service users, their families and practitioners. From reports with survivors and practitioners, we appreciate that a good deal of the pertinent evidence about interventions, and their benefits and harms, is missed from systematic reviews of trials. We also note that user and practitioner perspectives provide important information that may explain why interventions are not as effective, widely taken up or sustained in practice, compared to the controlled conditions in which they were tested. Hence, by conducting this QES to complement our review of trials, we aim to achieve comprehensive coverage of the evidence-base for effectiveness and also understand survivors', families' and practitioners' views of psychosocial interventions, with the intention of informing and improving future practice.

\section{How the intervention might work}

As described in more detail in Appendix 1 of our linked review (Brown 2019), the ways in which the interventions might work vary depending on the psychosocial intervention, the factors being targeted and the theoretical underpinnings and principles of the approach on which each intervention is founded. Some interventions are designed to be delivered within a short time period following the sexual abuse and violence (e.g. less than three months), whereas others are used for survivors in the longer term. The former attempt to provide prophylactic treatment to prevent chronic problems, while others intend to facilitate faster recovery (Vickerman 2009). CBT interventions are founded on the principles that behaviours are cognitively mediated (Butler 2006) and that cognitions (e.g. thinking patterns and beliefs) can be monitored and altered. Hence, behavioural change can be enacted via cognitive changes (Dobson 2009). Cognitive interventions for rape or sexual assault and trauma focus on two processes: (1) changing a person's cognitive appraisal of the traumatic event, or changing the process by which an individual attaches meaning to an event; and (2) changing a person's attribution of the event (Veronen 1983). Other cognitive interventions are designed to equip victims with coping skills to manage their trauma. Behavioural theorists argue that all behaviours are learned and unhealthy behaviours can be changed. Techniques such as flooding and systematic desensitisation are used to extinguish anxiety. Foa and colleagues believe, for example, that exposure to trauma allows mistaken evaluations and faulty stimulus-response associations to be corrected (Foa 1986). Victims are taught to replace a fear response with relaxation responses, which is done gradually in systematic desensitisation, and more quickly via flooding interventions. For example, in EMDR (Shapiro 1995), a survivor imagines a scene that represents the sexual abuse trauma and recites words related to it, while the therapist moves his/her fingers back and forth in front of the survivor, so that the survivor performs rhythmic, multisaccadic eye movements (quick, simultaneous movements of both eyes between two or more phases of fixation in the same direction) by watching the therapist's fingers. This movement is argued to facilitate the processing of trauma memory through the dual attention required to focus on attending to the therapist's finger movement (external stimulus) and the trauma scene (internal stimulus). However, studies comparing EMDR with and without eye movements show that EMDR without eye movements leads to equivalent outcomes as EDMR with eye movements (Boudewyns 1996; Pitman 1996). Many interventions combine behavioural and cognitive elements and hence are known as integrative therapies. For example, SIT (Veronen 1983), PET (Foa 1986) and CPT (Resnick 1997) all use combinations of relaxation training, flooding or systematic desensitisation techniques, psychoeducation and cognitive methods (see Brown 2019 for a more detailed outline of these approaches). Through acceptance, being present and committed action (Hayes 2006), third wave cognitive behavioural therapies, including mindfulness and acceptance and commitment therapy, act on changing the function of the events and the survivor's relationship to them. Counselling encompasses a range of interventions (Cryer 1980; Foa 1991; Resick 1988), premised on a number of theoretical schools of thought (e.g. humanist and psychodynamic). Counselling may be delivered alone or in combination with other approaches. Humanistic and supportive therapies include an eclectic mix of techniques. Supportive therapy is almost always non-directive, that is, the survivor is empowered to guide the content and the therapist avoids offering direct 
advice (Cohen 2005; Deblinger 2001). A diverse range of other psychologically-orientated interventions aim to help survivors cope with, express and work through trauma; for example, via expressive writing (Harte 2013), or the assistance of horses, which helps to reduce anxiety (Earles 2015). Psychosocial interventions are diverse and target a range of interpersonal, social and environmental factors in addition to, or instead of, the individual factors that are the focus of psychological therapies. Hence, the way these might work varies greatly. For example, psychoeducation aims to provide information, modelling and training. This might be to explain coping strategies and encourage the use of adaptive coping strategies over maladaptive ones (Sikkema 2018). Group programmes and the provision of advisors or mentors provide social support, which can be important given the stigma and shame associated with sexual abuse and violence that can lead to social isolation. These can increase self-esteem (Sikkema 2018), and provide emotional support and practical assistance (Home Office 2017).

Randomised controlled trials (RCTs) and meta-analyses for synthesising findings from several trials of interventions provide information on efficacy. They do not explain why some people might benefit and why others do not, or why some survivors might complete a treatment whilst others discontinue. The summary above, of how interventions might work, for example, shows that some interventions are driven by survivors, whereas others are led by professionals; some require survivors to recall the sexual abuse and violence, whereas others avoid or can be conducted without this. This is an important aspect, since there is reluctance for survivors or professionals (or both) to talk about the sexual abuse experienced prior to criminal justice trials for fear that this contaminates the survivor's testimony and undermines the court process. Even where there is a clear theoretical basis and hypothesis about the mechanism of change, RCTs cannot fully explicate the 'how' in the pathway. RCTs also assess effects based on a necessarily limited range of outcomes (and measures) and may fail to identify wider benefits and harms of the intervention. Thus, qualitative research is the ideal vehicle for answering these questions, as well as addressing questions around acceptability and for exploring the kinds of values and beliefs that might frame uptake of interventions. Data arising from qualitative studies can inform the content, delivery and provision of support for individuals who have experienced sexual abuse and violence, so that it is more effective, acceptable, accessible and of higher quality, particularly for marginalised or hard-to-reach groups. Hence, this QES will supplement our linked effectiveness review (Brown 2019), and may also extend interpretation of findings from related completed reviews (Gillies 2016; Macdonald 2012). .

\section{Why this review matters}

This QES is linked to a concurrent Cochrane Review of RCTs by an overlapping team of authors (Brown 2019), which addresses the evidence gap in our knowledge on the most effective ways of intervening to improve mental health outcomes for survivors of rape and sexual assault experienced during adulthood. It is also related to a review led by Caswell (Caswell 2019), one of the co-authors of this QES, assessing the measurement of patient experience and outcomes in healthcare settings after sexual violence. Additionally, it is related to evidence reviews conducted previously on interventions for children who have experienced CSA or trauma (or both) undertaken by different teams of authors, namely, Macdonald and colleagues' review evaluating cognitive-behavioural interventions for children who have been sexually abused (Macdonald 2012), and Gillies and colleagues' review of psychological therapies for children and adolescents exposed to trauma (Gillies 2016). While these reviews assess the evidence base for the effectiveness of interventions for survivors (adults and children) of sexual abuse and violence, or the ways in which survivors' experiences are measured, they do not assess survivors' and their families' and support networks' perspectives and experiences of these interventions, nor those of the professionals who deliver them. Hence, this QES will uncover mechanisms of intervention effectiveness, aiming to achieve a greater understanding of how and why an intervention might work or not. It will play a key role in developing a conceptual understanding of how different factors influence uptake, experiences, and dropout/completion of interventions from the perspective of survivors of sexual abuse and violence, their families and the professionals delivering the interventions. It is important to understand the experiences and views of these important stakeholder groups. We are aware from our practitioner partners, for example, that interventions and services are less frequently accessed by some groups (e.g. men; lesbian, gay, bisexual, and transgender (LGBT); ethnic minorities; and refugees) and that there are many barriers. For example, survivors have been denied access to interventions, particularly those that require talking about their experiences, for fear that this will change their memory or testimony and undermine the criminal trial process. Some interventions are not perceived positively by professionals and survivors, as they required extensive recall of the sexual abuse and violence, which is traumatic. It is therefore important to understand stakeholders' views and experiences of these interventions to gain a more complete understanding of the interventions' utility and accessibility.

Qualitative research can play a key role in developing our understanding about how interventions are experienced and work. Evidence from qualitative research and process evaluation studies can provide valuable insights into attitudes and perceptions of interventions, engagement, satisfaction, and barriers and facilitators experienced by stakeholders. It can also contribute to underlying mechanism(s) of change with regard to the particular intervention and the role of contextual factors in the delivery and impact of that intervention (Moore 2015; O'Doherty 2016). Understanding the views of intervention stakeholders who receive or deliver these interventions can help to inform decision-making and strategies regarding intervention development and enhancing their acceptability. The results from this QES, therefore, will enable us to have a greater understanding of context, benefits and harms of an intervention, and reasons for appropriateness, acceptability and implementation of interventions from the perspective of survivors, their families and professionals. Although we will not include quantitative process evaluation data, the synthesis of qualitative data may also contribute to understandings about mechanisms and pathways to change. Additionally, the results will enable us to examine how perceptions of an intervention may impact intervention engagement and effectiveness, and why intervention effects might vary across different contexts and subgroups. This may contribute to generating hypotheses about how and why certain interventions might be more effective for particular subgroups, and in which contexts, which is critical to informing subsequent subgroup analyses in future effectiveness reviews. Not all interventions available to survivors have been

Survivor, family and professional experiences of psychosocial interventions for sexual abuse and violence: a qualitative evidence 
evaluated using an RCT. Further, not all interventions, including those that have been tested in trials, will necessarily be perceived positively, and it is important to identify why interventions are not always widely taken up or sustained in practice compared to the controlled conditions in which they were tested. By conducting this QES to complement our systematic review of trials, we aim to achieve comprehensive coverage of the qualitative evidence base to understand the views of survivors, family members and practitioners, with the intention of informing and improving future practice. The findings may also help to inform the design of future trials, ensuring that they capture the elements of support that are important to survivors of sexual abuse and violence, their families and the professionals with whom they work.

\section{O B JECTIVES}

This review will gather and synthesise the experiences of interventions for survivors of sexual abuse and violence, their families, as well as the professionals who deliver them.

Specifically, this review seeks to:

1. identify, appraise and synthesise qualitative studies exploring the experiences of child and adult survivors of sexual abuse and violence, and their caregivers, regarding psychosocial interventions aimed at supporting survivors and preventing negative health outcomes in terms of benefits, risks/harms and barriers;

2. identify, appraise and synthesise qualitative studies exploring the experiences of professionals who deliver psychosocial interventions for sexual abuse and violence in terms of perceived benefits, risks/harms and barriers for survivors and their families/caregivers;

3. develop a conceptual understanding of how different factors influence uptake, dropout or completion, and outcomes from psychosocial interventions for sexual abuse and violence;

4. develop understanding of how features and types of interventions respond to the needs of different user/survivor groups (e.g. age groups; types of abuse exposure; migrant populations) and contexts (healthcare/therapeutic settings; low- and middle-income countries (LMICs)); and

5. explore how the findings of this review can enhance our understanding of the findings from the linked and related reviews assessing the effectiveness of interventions aimed at supporting survivors and preventing negative health outcomes.

\section{METHODS}

\section{Criteria for selecting studies for this review}

\section{Types of studies}

We will include primary empirical studies that are linked to a psychosocial intervention (as defined in the 'Types of interventions' section below) aimed at supporting survivors and preventing negative health outcomes that have:

1. qualitative study designs, such as ethnography, phenomenological studies, narrative studies, action research studies, case studies, grounded theory studies, visual studies and qualitative process evaluations;

2. both qualitative methods of data collection (e.g. focus group discussions, individual interviews, observation, diaries, arts- based methods, document analysis, and open-ended survey questions) and qualitative data analysis (e.g. thematic analysis, framework analysis, interpretative phenomenological analysis (IPA), grounded theory or other qualitatively inspired analytical approaches); and

3. mixed-methods designs where it is possible to extract the data that were collected and analysed using qualitative methods.

It is not a precondition that the published qualitative investigation be linked to studies included in the linked Cochrane Review and related reviews nor is it a criterion that they exist alongside a published evaluation of an intervention. For example, studies may focus on personal accounts of attending or receiving a type of intervention. Since we wish to stratify our findings across intervention types, we will include studies in which more than one intervention that meets our types of intervention criteria are examined, provided we can identify clearly the specific intervention to which each finding applies.

We will exclude:

1. studies that include data using qualitative methods but do not analyse these data using qualitative analysis methods (e.g. open-ended survey questions where the response data are analysed using descriptive statistics only); and

2. editorials, commentaries and opinion papers.

We will not exclude any studies based on our assessment of methodological limitations, but will utilise this information in our sampling strategy (see 'Selection of studies' section) and to assess our confidence in the synthesised findings.

\section{Types of participants}

Eligible studies will focus on at least one of three participant groups.

1. The primary group of interest is survivors of sexual abuse and violence. We will include studies that report on the experiences and views of people of any age, gender, sexuality or ethnicity who have received a psychosocial intervention in regards to experiencing sexual abuse or violence. We will include those who were offered an intervention, even if ultimately they did not take up or complete that intervention.

2. The review will also include studies focused on non-offending caregivers, parents and other family members in the context of a child or family member who is a victim of sexual abuse and violence, and is offered or receives a psychosocial intervention. This will allow us to gather the views, experiences, decisionmaking and acceptance of different psychosocial interventions for the individuals for whom the interventions are designed, but also to understand the views of those involved in the person's immediate support network.

3. The review will also include studies focused on providers involved in the direct delivery of the interventions (e.g. psychologists, counsellors, psychiatrists, support workers, ISVAs, and advocates).

We will exclude studies focused on policy makers, programme administrators, managers or other stakeholders. We will exclude studies related to interventions directed at family members or significant others.

Survivor, family and professional experiences of psychosocial interventions for sexual abuse and violence: a qualitative evidence 


\section{Subgroups}

As our review focuses on experiences of psychosocial interventions for different types of interventions, we will explore the similarities and differences in experiences across the different types of psychosocial interventions. In addition, where possible (i.e. where this information is discussed in the studies, or the characteristics of the participants voicing or experiencing different phenomena are clearly illustrated, or both), we will explore the similarities and differences in the experiences of different survivors (e.g. children or adults, gender, ethnicity and sexuality, types of abuse), contexts (urban or rural, high-income country, low- or middleincome country), family members or caregivers (e.g. mother or father or other caregiver, gender and ethnicity), clinical outcomes (e.g. type of clinical outcome, impact of the intervention for the individual), intervention completion (e.g. completers, noncompleters, dropouts) and professionals (e.g. profession, levels of experience or training, gender and ethnicity).

\section{Settings}

Any setting where a person has received, or a professional has delivered, an intervention aimed at providing psychosocial support to a survivor in the aftermath of experiencing any form of sexual abuse or sexual violence. Settings could include health care, such as general practice, sexual health and mental health services; schoolbased/education services; charity and voluntary sector services in the mental health or sexual and domestic violence sectors; local support communities, and home support programmes (e.g. home visits).

\section{Types of interventions}

The intervention consists of any type of psychosocial intervention that targets recovery from sexual abuse or sexual violence and that meets the definition of "interpersonal or informational activities, techniques, or strategies that target biological, behavioral, cognitive, emotional, interpersonal, social, or environmental factors with the aim of improving health functioning and wellbeing" (Committee on Developing Evidence-Based Standards for Psychosocial Interventions for Mental Disorders (IOM 2015)), including the following.

1. Formal CBT, TF-CBT and CBT-based techniques.

2. Integrative therapies, including SIT (Veronen 1983), and later, PET (Foa 1986) and CPT (Resnick 1997).

3. Behaviour therapies such as EMDR and relaxation techniques.

4. Third wave CBT (e.g. acceptance and commitment Therapy, mindfulness)

5. Humanistic therapies (e.g. supportive and non-directive therapy).

6. Other psychologically-orientated interventions (e.g. art therapy, meditation, trauma-informed body-based practices (e.g. embodied relational therapy, yoga and Tai Chi), narrative therapy).

7. Other psychosocial interventions, including support services delivered by mentors, support workers, advisors or advocates such as ISVAs in the UK, support groups, and coping interventions.

We will include interventions of any duration or frequency of treatment so long as the intervention meets the criteria stated above.
Intervention delivery could be face-to-face, by telephone, through computer-based systems or by any combination of these delivery modes.

We will include both individual and group delivered interventions. It will need to be clear what intervention type has been experienced by the participant.

\section{Types of phenomena of interest}

The topic of interest in this synthesis is the factors (e.g. contextual and individual) involved in uptake and continuance of treatment for exposure to sexual abuse and violence across the lifespan; the experience of receiving (and providing) an intervention or treatment; and the benefits and risks/harms for survivors and their families/caregivers from both their perspectives and the perspectives of the professionals involved in providing such interventions.

We will explore the following phenomena.

1. The factors involved in the uptake and continuance of treatment for exposure to sexual abuse and violence at any age (short-term phenomena).

2. Survivors', caregivers' or families' and professionals' beliefs, attitudes, perceptions and experiences of the intervention (medium-term phenomena).

3. Survivors', caregivers' or families' and professionals' reported short, medium and long-term benefits and barriers, as well as risks/harms following exposure to interventions.

The type of evidence collected in our synthesis will include also participants' satisfaction with components of a support programme provided; for instance, in terms of level of training, and demographic and professional characteristics of the providers.

\section{Search methods for identification of studies}

The search methods for this review have been developed using guidelines published by the Cochrane Qualitative and Implementation Methods Group (CQIMG) (Harris 2018, including their earlier guidance (Booth 2011)). We will organise the search into two strands, adding a third if necessary, to allow for additional searches to capture specific themes that we uncover during the screening process and that we want to explore in more depth.

Strand 1 will identify qualitative evidence in reports related to, or embedded in, RCTs identified in the linked effectiveness review (Brown 2019) and in other relevant systematic reviews of which we are aware (Caswell 2019; Gillies 2016; Macdonald 2012). We will use the same approach for other systematic reviews that we identify during other phases of the search. Strand 2 will use a broad systematic search, including databases from a range of disciplines, and supplementary searches to increase the chance of finding eligible studies not indexed in bibliographic databases, or that do not contain the search terms in our core search strategy. In Strand 3 , we will examine any eligible studies found in Strands 1 and 2, and will assess whether they fulfil our aims, objectives and criteria for this review. If necessary, we will revise our search terms and conduct additional searches. 


\section{Strand 1}

We will use the search results from the linked effectiveness review (Brown 2019) to identify qualitative evidence embedded in reports of trials, and additionally, will identify qualitative studies associated with related systematic reviews, including three originally used to establish a rationale for undertaking the linked review. These related reviews examined the evidence for cognitive-behavioural interventions for children who have been sexually abused (Macdonald 2012), psychological therapies for children and adolescents exposed to trauma (Gillies 2016), and the measurement of patient experience and outcome in health care settings on receiving care after sexual violence (Caswell 2019). We will try also to contact the principal trial investigators of studies included in these reviews and the authors of the three related reviews (note, Caswell is an author of this QES) to ask about the existence of studies that meet the criteria for this qualitative review (Noyes 2019). We will include any studies identified in the search as meeting the criteria for this QES, even if they are excluded in the linked review (Noyes 2019).

Next, we will extract authors' names and keywords from the titles and abstracts of the quantitative studies in both the linked and related reviews, and use them to search for separate reports of qualitative data related to the trials (Booth 2011; Booth 2013).

We will adopt the same approach for relevant systematic reviews identified in other phases of the search.

\section{Strand 2}

\section{Database searches}

We will conduct a systematic search for this QES (independent of the linked effectiveness review (Baumeister 2019)), using the databases listed below.

1. MEDLINE Ovid (1946 onwards)

2. MEDLINE Ovid, Epub Ahead of Print (current issue)

3. MEDLINE Ovid, In-Process \& Other Non-indexed Citations (current issue)

4. Embase Ovid (1974 onwards)

5. CINAHL Plus EBSCOhost (Cumulative Index to Nursing and Allied Health Literature; 1937 onwards)

6. PsycINFO Ovid (1806 onwards)

7. Epistemonikos (www.epistemonikos.org/en; current issue, limited to systematic reviews)

8. PDQ-Evidence (www.pdq-evidence.org; current issue)

9. Social Services Abstracts Proquest (1979 onwards)

10.Social Science Citation Index Clarivate Web of Science (1970 onwards)

11.PTSDpubs Proquest (1871 onwards)

12.ProQuest Dissertations \& Theses A\&I Proquest (1743 onwards)

We will search MEDLINE using a search strategy adapted from the linked evaluation review (Brown 2019), and in consultation with the Cochrane Information Specialist for Developmental, Psychosocial and Learning Problems. We will expand the population section to include search terms for children who have been sexually abused (the evaluation review is limited to sexual abuse in adulthood). The sensitivity of the intervention section will be augmented with general terms for treatment because qualitative studies may not necessarily refer to specific psychosocial interventions. Where possible, we will replace the filters used to find RCTs in the linked evaluation review with published filters to find qualitative studies (McKibbon 2006; Walters 2006; Wilczynski 2007; Wong 2004), revising them as necessary to reflect, for example, new indexing terms (Appendix 1).

\section{Supplementary searches}

\section{Reference lists}

We will examine the reference lists of all included studies in this QES and in the linked or related reviews.

\section{Related references}

We will conduct a forward citation search using the Social Science Citation Index of included studies in this QES and in the linked or related reviews.

\section{Correspondence}

In addition to contacting authors of all included studies and related reviews, we will contact experts in this field to identify studies that meet our criteria, including unpublished and ongoing research.

\section{Unpublished reports}

As we believe that qualitative studies of user and practitioner perspectives may not all be included in bibliographic databases, we will also search for unpublished reports. We will search national (e.g. Women's Aid and Rape Crisis) and international websites (e.g. World Health Organization and United Nations Preventing Sexual Exploitation and Abuse) and Sexual Assault Referral Centre websites (e.g. Blue Sky Centre and Juniper Lodge) in the UK, in addition to the following sources.

1. National Institute for Health Research search portal (www.nihr.ac.uk/health-and-care-professionals/searchour-evidence.htm)

2. OpenGrey (www.opengrey.eu)

3. Grey Literature Report (www.nyam.org/library/collections-andresources/grey-literature-report)

\section{Strand 3}

We will examine eligible studies identified during Strands 1 and 2 . If we find ideas or themes that we want to explore in more depth, we will conduct specific searches using new search terms with the help of our information specialist, until we are confident that we have searched for all relevant key terms and that our electronic searches have identified as many of the qualitative studies that meet our criteria as is feasible within the time and resources available for the review.

\section{Data collection and analysis}

\section{Selection of studies}

\section{Screening phase}

Two review authors (NK and GC) will independently assess the titles and abstracts of all records identified through the literature searches against criteria for considering studies for this QES. They will code abstracts as 'retrieve' (eligible, potentially eligible or unclear) or 'do not retrieve' (not eligible). In the event of disagreements about inclusion, both review authors will assess 
and discuss the full article for relevance, using the GRADECERQual (Confidence in the Evidence from Reviews of Qualitative research) approach (Noyes 2018). If agreement cannot be reached by discussion, they will consult a third review author (SB) as a mediator. Final decisions will be made by consensus. We will retrieve full-text articles for selected abstracts and two pairs of review authors (GC and SB, EH and LOD) will independently assess each article against the criteria for considering studies for this QES. Studies will be identified for either inclusion or exclusion. We will contact study authors, as required, to decide whether the inclusion criteria have been met. We will record reasons for excluding ineligible studies. In the event of disagreements, we will consult a third review author (KB) as a mediator. Final decisions will be made by consensus.

For titles and abstracts that are published in a language in which none of the review team and colleagues are fluent, we will carry out an initial translation through open source software (Google Translate). If this translation indicates inclusion, or if the translation is inadequate to make a decision, we will retrieve the full text of the paper. We will then ask members of Cochrane networks or other networks that are fluent in that language to assist us in assessing the eligibility of the full text of the paper for inclusion. If this cannot be done for a paper in a particular language, we will list the paper as 'studies awaiting classification', to ensure transparency in the review process. If we decide to include studies published in languages in which the review team are not fluent, we will obtain a professionally translated version of the entire paper.

We will identify and exclude duplicate records, and will collate multiple reports that relate to the same study so that each study rather than each report is the unit of interest in the review. We will record the selection process in sufficient detail to complete a four-phase (identification, screening, eligibility and included) PRISMA flow diagram for study collection (Moher 2009), and the 'Characteristics of excluded studies' Tables.

\section{Sampling framework}

Including every qualitative study we find that meets our criteria in this QES threatens its quality because it is time-consuming and will prevent us from completing an in-depth exploration of our aims and objectives. Furthemore, exhaustive sampling risks producing "superficial synthesis findings, with a large number of studies that fail to go beyond the level of description" (Benoot 2016). Therefore, we will employ Benoot's "umbrella approach" (Benoot 2016), combining several purposeful sampling techniques.

We will begin by reviewing the articles identified as meeting our QES criteria (criterion sampling, Suri 2011), conducting preparatory data extraction in order to identify the type and range of psychosocial interventions that have been examined; the sampling across our three groups of survivors, families/carers and professionals; and the characteristics (e.g. gender, age, ethnicity, sexuality) of these samples. This information will be collated and used to develop a stratified purposive sample (Suri 2011). This is to ensure that we can explore the views and experiences of our three groups across the range of psychosocial interventions while taking account, if possible, of participant characteristics and study context, in line with our QES aims and objectives. We will use a maximum variation strategy (Suri 2011), to ensure that we have considered the benefits, risks/harms and barriers for diverse populations. At this stage, we will include studies with overlapping samples or characteristics, and complete full data extraction and an appraisal of the quality of each study.

The final selection of studies for each of our stratified criteria will be based on the availability of relevant information (e.g. participant characteristics) and the quality of studies (i.e. selecting studies rated as high quality and with more complete information). Where possible, given the availability of studies, we will include one study that examines each of the three groups (survivors, families or caregivers, and professionals) for each of the psychosocial interventions examined, and for each of our participant characteristics types (which will be determined on the basis of variation in the samples in the available range of studies).

\section{Data extraction and management}

We will perform data extraction using a form designed specifically for this review. We will extract information on: first study author; date of publication; country of study; context (urban or rural; high-income country, low- or middle-income country); type of intervention along with duration and details about the locations or settings; participant groups (survivors, families/carers, professionals); type of abuse experienced by participants; sampling strategy and ethical considerations; number of participants in each group; participants' age, gender, sexuality and ethnicity; data collection methods and justification for these; data analysis methods; key findings and the extent to which findings are supported by sufficient evidence; and details of reflexivity. We will extract relevant findings, including extracts from participants and authors, themes and sub-themes, explanations, hypotheses, theories, observations and interpretations of these data (Sandelowski 2002), reported anywhere in the primary qualitative studies. As the thematic synthesis approach shares characteristics with meta-ethnography and grounded theory (Barnett-Page 2009), we will use the same approach to identify findings regardless of the qualitative approach or analyses (e.g. thematic analysis, grounded theory) used in the study. We will use EPPI-Reviewer 4 (Thomas 2008; Thomas 2010) as a platform to manage our screening process, upload search results, select studies, extract and record data, resolve disagreements, export data into Review Manager 5 (Review Manager 2014), and generate a PRISMA diagram report.

\section{Appraisal of methodological limitations in the included studies}

We will only include studies that have a transparent audit trail of the methods used, which is a basic quality threshold. We will assess the quality of each study using an adapted version of the Critical Appraisal Skills Programme (CASP) tool (Atkins 2008; CASP 2018), which has been used in other reviews and protocols of qualitative evidence syntheses (Ames 2017; Carlsen 2016; Houghton 2017).

The adapted tool includes the following questions, which we will use to assess methodological limitations.

1. Is the qualitative research approach appropriate for the research question?

2. Is the qualitative research approach stated clearly?

3. Is the qualitative research approach justified clearly?

4. Are ethical issues considered and is formal ethical approval granted?

5. Is the sampling method described clearly?

6. Is the sampling method appropriate for the research question?

Survivor, family and professional experiences of psychosocial interventions for sexual abuse and violence: a qualitative evidence 
7. Is the method of data collection appropriate for the research question?

8. Does the approach to data analysis address the research question?

9. Is the approach to data analysis described clearly?

10.Are the researcher's findings supported by sufficient evidence?

Two review authors with qualitative research experience (SB and GC) will independently assess each study. Disagreements will be resolved through discussion and consultation of a third author (RC). As it is recognised that studies deemed to be of low quality may still provide new insights (Dixon-Woods 2005; Noyes 2019), we will not exclude studies on the basis of quality although as described previously, we will use the quality of the study in our purposeful stratified sampling strategy. As suggested by Hannes 2011, appraisals of the methodological limitations of the studies will form part of the assessment of confidence in the synthesis findings (discussed below) using the GRADE-CERQual assessment (Lewin 2018), which determines the level of confidence we can have in each finding in the synthesis.

\section{Assessment of confidence in the synthesis findings}

We will apply the GRADE-CERQual approach (Lewin 2018), to assess the level of confidence to place in individual review findings. Two review authors (SB and GC) will independently summarise our confidence in each finding, with disagreements resolved through discussion and consultation with a third review author (EH). GRADE-CERQual provides a transparent and structured method for assessing confidence in the findings of qualitative syntheses. The tool focuses on the following four components that assess how much confidence to place in an individual finding.

1. Methodological limitations of included studies: The extent to which there are concerns about the design or conduct of the primary studies that contributed evidence to an individual review finding.

2. Coherence of the review finding: The extent to which the review finding is well grounded in data from the contributing primary studies and provides a convincing explanation for the patterns found in these data.

3. Adequacy of the data contributing to a review finding: An overall determination of the degree of richness and quantity of data supporting a review finding.

4. Relevance of the included studies to the review question: The extent to which the body of evidence from the primary studies supporting a review finding is applicable to the context (perspective or population, phenomenon of interest, setting) specified in the review question.

We will generate a 'CERQual Evidence Profile' for each finding. This will include information on all CERQual component assessments, which we will use to make an overall judgement of confidence. All findings will be rated at high confidence initially and then graded down when there are important concerns regarding each of the CERQual components. The assessment will be discussed and agreed by SB and GC and quality assured by EH. Each finding, therefore, will be graded at one of the following four levels:

1. high confidence, where it is highly likely that the review finding is a reasonable representation of the phenomenon of interest;
2. moderate confidence, where it is likely that the review finding is a reasonable representation of the phenomenon of interest;

3. low confidence, where it is possible that the review finding is a reasonable representation of the phenomenon of interest; and

4. very low confidence, where it is not clear whether the review finding is a reasonable representation of the phenomenon of interest (Lewin 2018).

We will produce a 'Summary of qualitative findings' table to present key findings, including our overall CERQual assessment for each finding. We will follow the methodological guidance on creating an evidence profile and 'Summary of qualitative findings' table provided by the CERQual working group, and as illustrated and described in Lewin 2018. We will present detailed descriptions of our confidence assessment in an Evidence Profile(s).

\section{Data synthesis}

In the first instance, we will synthesise data using a thematic methodology, to identify the themes and alternative theories that exist throughout the studies (Thomas 2008). The process of synthesising qualitative evidence involves the comparison and analysis of findings from a variety of sources (Noyes 2019). The purpose of this method is to develop analytical themes through a descriptive synthesis and to find explanations relevant to the review questions (Ring 2011). We will use EPPI-Reviewer (Thomas 2010), specialist reviewing software, for this analysis. If this is not a good fit for this QES, then we will consider other, more appropriate synthesis methods.

The thematic synthesis, if a good fit for this QES, will include three overlapping stages. First, one review author will conduct free, lineby-line coding to identify first order constructs, that is, primary data such as quotes or other data such as images, and second order constructs, that is, themes and sub-themes generated by the authors of the studies. A second author will review a $10 \%$ subsample to quality assure the synthesis. We will include verbatim extracts in the report to illustrate the findings. At this stage, we will set aside our review questions in order to adopt an inductive, datafocused approach. This will avoid imposing an a priori framework onto the findings without allowing for the possibility that a different framework will represent the data better (Thomas 2008). This will generate a set of first order codes and second order constructs that will be increased and developed as each study is coded. When all studies have been coded, we will examine all of the text that have been given a code, code by code, in order to check for consistency and to identify if additional codes are needed (Thomas 2008).

For the second stage, we will compare the first and second order constructs coded in stage one against the review questions, and examine each study again in order to identify more abstract interpretations. Our starting point will be a collection of studies, using stratified purposeful followed by maximum variation sampling, as described previously. We will then take a more indepth approach to synthesising and analysing the similarities and variances between the themes and concepts evident in the studies. We will do this by collectively examining the similarities and differences between the first and second order constructs to begin to organise them into descriptive themes. Where necessary, we will develop new second order constructs to capture the meaning of groups of extracted first and second order constructs (Thomas 2008). We will review and discuss the constructs until we have developed an appropriate order, structure or framework that best 
presents the themes and allows us to synthesis them with the findings from the linked and related reviews. Until this point, the synthesis produced will stay close to the primary findings in the included studies.

The third analytical synthesis stage will move beyond the findings in the primary studies to develop new patterns, meanings and understandings. We will then integrate the findings from the linked (Brown 2019) and related reviews (Gillies 2016; Macdonald 2012), described in the section below. We will conduct a sequential synthesis using the related and linked studies (Harden 2018). Although we are conducting the linked review and QES in a similar time frame, we are completing the linked review a stage ahead of the QES. Hence, we will be able to integrate the findings of the reviews, once we have completed the analysis for the linked review and understand what that tells us about the effectiveness of interventions. These findings will allow us to generate another set of questions to further develop our third order constructs as we report new interpretations of the integrated data.

\section{Using the synthesised qualitative findings to supplement the Cochrane Reviews on effectiveness}

Our aim in conducting this QES is to identify and draw together the experiences of those delivering and receiving psychosocial interventions, allowing us to supplement our linked review (Brown 2019) and add to others (Gillies 2016; Macdonald 2012), by increasing understanding about the benefits, lack of benefit and harms of interventions. For example, we are aware from our practitioner partners that there is not necessarily a match between what is evaluated as part of RCTs and what practitioners are using in practice; interventions that show benefits in trials are not necessarily viewed as helpful by users; and conversely, negative trials may generate benefits not detected in those trials. Hence, the findings of this review, stratified by types of interventions, child or adult survivor (and other characteristics such as gender, ethnicity and sexuality), and families' and professionals' experiences will provide important information for practice on people's experiences of interventions, including their perceived benefits, risks/harms and barriers, as well as the appropriateness and acceptability of interventions for implementation. We will make a final decision on our integration method when we have completed the linked review and have a full understanding of the effects of the intervention, and when the data synthesis for this QES is completed. One possibility, if there are differences across interventions and/or participants and contexts, is that we will juxtapose findings in a matrix (Harden 2018). If there is more homogeneity in the findings, we will create a conceptual model to present this information to supplement the linked review and potentially the related reviews (Harden 2018). Using this approach, we aim to highlight interventions that have demonstrated both effectiveness, based on trial outcomes, and are also viewed positively by stakeholder groups. We will also identify areas where it might be possible to enhance user experiences, address potential harms and minimise barriers to uptake, based on the synthesised feedback across the stakeholder groups.

\section{Sensitivity analysis}

We will undertake a sensitivity analysis if low-quality studies affect the conceptual model. This will help us assess how much of an impact these studies have on our analysis and findings, as suggested by Dixon-Woods and colleagues (Dixon-Woods 2006). Following our thematic synthesis, we will examine the contributions to the analytic themes and subsequent interventions (Thomas 2008).

\section{Researchers' reflexivity}

All authors of this QES and of the larger MESARCH (Multidisciplinary Evaluation of Sexual Assault Referral Centres for better Health) study believe in the importance of supporting and empowering all victims of sexual abuse and violence. Our QES and MESARCH teams comprise of academics from different disciplines and perspectives, practitioners, commissioners, policymakers and individuals with lived experiences of sexual abuse and violence. The MESARCH project includes a Lived Experience Group and a Study Steering Committee, and members regularly review progress and drafts of materials and reports, including this QES protocol. We hope that these teams, representing a wide range of perspectives, experiences, contexts and backgrounds, and the regular review processes by the different groups will encourage reflexivity, as well as ensuring that practitioner and lived experiences perspectives are represented. Our work to date and hence forth, will be discussed regularly among the QES and MESARCH teams to review progress, clarify procedures or methods, and identify and challenge assumptions. In our data analysis and in synthesising the findings of the QES and integrating these with the linked or related reviews, the authors conducting the analyses will use refutational analysis techniques ('disconfirming analyses'), to explore and try to understand contradictory findings between studies. These analyses will be reviewed by other members of the QES team and wider MESARCH group as per its review processes. We will document these measures in the reflexivity section of the report.

\section{ACKNOWLEDGEMENTS}

This research is funded by an institutional (Coventry University) research grant (project number 16/117/04: Multidisciplinary Evaluation of Sexual Assault Referral Centres for better Health (MESARCH)) from the National Institute for Health Research (NIHR) Health Service and Delivery Research (HS\&DR) Programme. The views expressed herein are those of the authors and not necessarily those of the NIHR, NHS, or the Department of Health.

We would like to thank members of the MESARCH Study Steering Committee and our Lived Experiences Group for their input into the development of this protocol.

We thank Associate Professor Margaret Cargo and Professor Jane Noyes, both of the Cochrane Qualitative and Implementation methods group, and members of the Cochrane Developmental, Psychosocial and Learning Problems review group for their guidance in developing this protocol; as well as the following reviewers who commented on earlier versions: Anao Zhang, PhD, University of Michigan; Lisa Fedina, PhD, University of Michigan; and Dee Shneiderman. 


\section{R E F E R E N C E S}

\section{Additional references}

\section{Ames 2017}

Ames HM, Glenton C, Lewin S. Parents' and informal caregivers' views and experiences of communication about routine childhood vaccination: a synthesis of qualitative evidence. Cochrane Database of Systematic Reviews 2017, Issue 2. [DOI: 10.1002/14651858.CD011787.pub2] [PMC5461870] [PMID: 28169420]

\section{Atkins 2008}

Atkins S, Lewin S, Smith H, Engel M, Fretheim A, Volmink J. Conducting a meta-ethnography of qualitative literature: lessons learnt. BMC Medical Research Methodology 2008; 8:21. [DOI: 10.1186/1471-2288-8-21] [PMC2374791 ] [PMID: 18416812]

\section{Barnett-Page 2009}

Barnett-Page E, Thomas J. Methods for the synthesis of qualitative research: a critical review. BMC Medical Research Methodology 2009; 9:59. [DOI: 10.1186/1471-2288-9-59] [PMC3224695 ] [PMID: 19671152]

\section{Baumeister 2019}

Bohren MA, Berger BO, Munthe-Kaas H, . Perceptions and experiences of labour companionship: a qualitative evidence synthesis. Cochrane Database of Systematic Reviews 2019, Issue 3. [DOI: 10.1002/14651858.CD012449.pub2] [PMC6422112] [PMID: 30883666]

\section{Benoot 2016}

Benoot C, Hannes K, Bilsen J. The use of purposeful sampling in a qualitative evidence synthesis: a worked example on sexual adjustment to a cancer trajectory. BMC Medical Research Methodology 2016; 16:21. [DOI: 10.1186/ s12874-016-0114-6] [PMC4757966] [PMID: 26891718]

\section{Booth 2011}

Booth A. Chapter 3: Searching for Studies. In: Noyes J, Booth A, Hannes K, Harden A, Harris J, Lewin S, Lockwood C (editors), Supplementary Guidance for Inclusion of Qualitative Research in Cochrane Systematic Reviews of Interventions. Version 1 (updated August 2011). Cochrane Collaboration Qualitative Methods Group, 2011. Available from cqrmg.cochrane.org/ supplemental-handbook-guidance.

\section{Booth 2013}

Booth A, Harris J, Croot E, Springett J, Campbell F, Wilkins E. Towards a methodology for cluster searching to provide conceptual and contextual "richness" for systematic reviews of complex interventions: case study (CLUSTER). BMC Medical Research Methodology 2013; 13:118. [DOI: 10.1186/1471-2288-13-118] [PMC3819734 ] [PMID: 24073615]

\section{Boudewyns 1996}

Boudewyns PA, Hyer LA. Eye movement desensitization and reprocessing (EMDR) as treatment for post-traumatic stress disorder (PTSD). Clinical Psychology \& Psychotherapy 1996; 3(3):185-95. [DOI: 10.1002/(SICI)1099-0879(199609)3:3<185::AIDCPP101>3.0.CO;2-0 ]

\section{Brennan 2012}

Brennan J, Kuhns LM, Johnson AK, Belzer M, Wilson EC, Garofalo R, et al. Syndemic theory and HIV-related risk among young transgender women: the role of multiple, co-occurring health problems and social marginalization. American Journal of Public Health 2012; 102(9):1751-7. [DOI: 10.2105/ajph.2011. 300433] [PMC3416048 ] [PMID: 22873480]

\section{Brown 2019}

Brown SJ, Khasteganan N, Brown K, Hegarty K, Carter GJ, Tarzia L, et al. Psychosocial interventions for survivors of rape and sexual assault experienced during adulthood. Cochrane Database of Systematic Reviews 2019, Issue 11. [DOI: 10.1002/14651858.CD013456

\section{Burgess 1974}

Burgess AW, Holmstrom LL. Rape trauma syndrome. American Journal of Psychiatry 1974; 131(9):981-6. [DOI: 10.1176/ ajp.131.9.981]

\section{Butler 2006}

Butler AC, Chapman JE, Forman EM, Beck AT. The empirical status of cognitive-behavioral therapy: a review of metaanalyses. Clinical Psychology Review 2006; 26(1):17-31. [DOI: 10.1016/j.cpr.2005.07.003] [PMID: 16199119]

\section{Campbell 1998}

Campbell R. The community response to rape: victims' experiences with the legal, medical, and mental health systems. American Journal of Community Psychology 1998; 26(3):355-79. [DOI: 10.1023/a:1022155003633] [PMID: 9726113]

\section{Campbell 1999}

Campbell JC, Soeken KL. Forced sex and intimate partner violence: effects on women's risk and women's health. Violence Against Women 1999; 5(9):1017-35. [DOI: 10.1177/1077801299005009003]

\section{Campbell 2001}

Campbell R, Martin PY. Services for sexual assault survivors: the role of rape crisis centers. In: Renzetti CM, Edleson JL, Kennedy Bergen R, editors(s). Sourcebook on Violence Against Women. Thousand Oaks (CA): Sage Publications, Inc, 2001:227-41.

\section{Campbell 2004}

Campbell R, Sefl T, Ahrens CE. The impact of rape on women's sexual health risk behaviours. Health Psychology 2004; 23(1):67-74. [DOI: 10.1037/0278-6133.23.1.67] [PMID: 14756605]

\section{Carlsen 2016}

Carlsen B, Glenton C. The swine flu vaccine, public attitudes, and researcher interpretations: a systematic review of qualitative research. BMC Health Services Research 2016; 16:203. [DOI: 10.1186/s12913-016-1466-7] [PMC4919843 ] [bmchealthservres.biomedcentral.com/articles/10.1186/ s12913-016-1466-7] [PMID: 27338141] 


\section{CASP 2018}

Critical Appraisal Skills Programme. CASP qualitative checklist. casp-uk.net/wp-content/uploads/2018/01/CASP-QualitativeChecklist-2018.pdf (accessed 24 July 2019).

\section{Caswell 2019}

Caswell RJ, Ross JD, Lorimer K. Measuring experience and outcomes in patients reporting sexual violence who attend a healthcare setting: a systematic review. Sexually Transmitted Infections 2019; 95(6):419-27. [DOI: 10.1136/sextrans-2018-053920] [PROSPERO registration: RD42016050297] [PMID: 31217323]

\section{CDC 2004}

Centers for Disease Control and Prevention (CDC). Sexual Violence Prevention: Beginning the Dialogue. Altanta (GA): Centers for Disease Control and Prevention, 2004.

\section{Cohen 2005}

Cohen JA, Mannarino AP, Knudsen K. Treating sexually abused children: 1 year follow-up of a randomised controlled trial. Child Abuse \& Neglect 2005; 29(2):135-45. [DOI: 10.1016/ j.chiabu.2004.12.005] [PMID: 15734179]

\section{CPS 2002}

, Department of Health and Home Office. Provision of Therapy for Vulnerable or Intimidated Witnesses Prior to a Criminal Trial: Practice Guidance. London (UK): Home Office Communications Directorate, 2002.

\section{Cryer 1980}

Cryer L, Beutler L. Group therapy: an alternative treatment approach for rape victims. Journal of Sex \& Marital Therapy 1980; 6(1):40-6. [DOI: 10.1080/00926238008404244] [PMID: 7381947]

\section{Deblinger 2001}

Deblinger E, Stauffer LB, Steer RA. Comparative efficacies of supportive and cognitive behavioral group therapies for young children who have been sexually abused and their nonoffending mothers. Child Maltreatment 2001; 6(4):332-43. [DOI: 10.1177/1077559501006004006] [PMID: 11675816]

\section{Dixon-Woods 2006}

Dixon-Woods M, Bonas S, Booth A, Jones DR, Miller T, Sutton AJ, et al. How can systematic reviews incorporate qualitative research? A critical perspective. Qualitative Research 2006; 6(1):27-44. [DOI: 10.1177/1468794106058867]

\section{Dixon-Woods 2005}

Dixon-Woods M, Agarwal S, Jones D, Young B, Sutton A. Synthesising qualitative and quantitative evidence: a review of possible methods. Journal of Health Services Research \& Policy 2005; 10(1):45-53. [DOI: org/10.1177/135581960501000110] [PMID: 15667704 ]

\section{Dobson 2009}

Dobson KS. Handbook of Cognitive-Behavioral Therapies. 3rd edition. New York (NY): Guilford Press, 2009.

\section{Dognin 2017}

Dognin J, Sedlander E, Jay M, Ades V. Group education sessions for women veterans who experienced sexual violence: qualitative findings. Families, Systems \& Health 2017; 35(3):360-72. [DOI: 10.1037/fsh0000262] [PMID: 28414479]

\section{Dubourg 2005}

Dubourg R, Hamed J, Thorns J. The Economic and Social Costs of Crime Against Individuals and Households 2003/04. London (UK): Home Office, 2005. [webarchive.nationalarchives.gov.uk/20100408132849/http:// www.homeoffice.gov.uk/rds/pdfs05/rdsolr3005.pdf]

\section{Earles 2015}

Earles JL, Vernon LL, Yetz JP. Equine-assisted therapy for anxiety and posttraumatic stress symptoms. Journal of Traumatic Stress 2015; 28(2):149-52. [DOI: 10.1002/jts.21990] [PMID: 25782709]

\section{Foa 1986}

Foa EB, Kozak MJ. Emotional processing of fear: exposure to corrective information. Psychological Bulletin 1986; 99(1):20-35. [PMID: 2871574]

\section{Foa 1991}

Foa EB, Rothbaum BO, Riggs DS, Murdock TB. Treatment of posttraumatic stress disorder in rape victims: a comparison between cognitive behavioral procedures and counseling. Journal of Consulting and Clinical Psychology 1991; 59(5):715-23. [DOI: 10.1037//0022-006x.59.5.715] [PMID: 1955605]

\section{Ford 2010}

Ford JD, Elhai JD, Connor DF, Frueh BC. Poly-victimization and risk of posttraumatic, depressive, and substance use disorders and involvement in delinquency in a national sample of adolescents. Journal of Adolescent Health 2010; 46(6):545-52. [DOI: 10.1016/j.jadohealth.2009.11.212] [PMID: 20472211]

\section{Gillies 2016}

Gillies D, Maiocchi L, Bhandari AP, Taylor F, Gray C, O'Brien L. Psychological therapies for children and adolescents exposed to trauma. Cochrane Database of Systematic Reviews 2016, Issue 10. [DOI: 10.1002/14651858.CD012371] [PMC6457979] [PMID: 27726123]

\section{Goodman 1993}

Goodman LA, Koss MP, Russo NF. Violence against women: physical and mental health effects. Part I: research findings. Applied and Preventative Psychology 1993; 2(2):79-89. [DOI: 10.1016/S0962-1849(05)80114-3]

\section{Guina 2019}

Guina J, Nahhas RW, Kawalec K, Farnsworth S. Are gender differences in DSM-5 PTSD symptomatology explained by sexual trauma? Journal of Interpersonal Violence 2019; 34(21-22):4713-40. [DOI: 10.1177/0886260516677290] [PMID: 27827321]

\section{Hannes 2011}

Hannes K. Chapter 4: Critical appraisal of qualitative research. In: Noyes J, Booth A, Hannes K, Harden A, Harris J, Lewin S, 
Lockwood C (editors), Supplementary Guidance for Inclusion of Qualitative Research in Cochrane Systematic Reviews of Interventions. Version 1 (updated August 2011). Cochrane Collaboration Qualitative Methods Group, 2011. Available from cqrmg.cochrane.org/supplemental-handbook-guidance.

\section{Harden 2018}

Harden A, Thomas J, Cargo M, Harris J, Pantoja T, Flemming K, et al. Cochrane Qualitative and Implementation Methods Group guidance series-paper 5: methods for integrating qualitative and implementation evidence within intervention effectiveness reviews. Journal of Clinical Epidemiology 2018; 97:70-8. [DOI: 10.1016/j.jclinepi.2017.11.029] [PMID: 29242095]

\section{Harris 2018}

Harris JL, Booth A, Cargo M, Hannes K, Harden A, Flemming K, et al. Cochrane Qualitative and Implementation Methods Group guidance series-paper 2: methods for question formulation, searching, and protocol development for qualitative evidence synthesis. Journal of Clinical Epidemiology 2018; 97:39-48. [DOI: 10.1016/j.jclinepi.2017.10.023] [PMID: 29248725]

\section{Harte 2013}

Harte CB, Hamilton LD, Meston CM. Predictors of attrition from an expressive writing intervention for sexual abuse survivors. Journal of Child Sexual Abuse 2013; 22(7):842-57. [DOI: 10.1080/10538712.2013.830670] [PMID: 24125085]

\section{Harvey 1996}

Harvey MR. An ecological view of psychological trauma and trauma recovery. Journal of Traumatic Stress 1996; 9(1):3-23. [PMID: 8750448]

\section{Hayes 2006}

Hayes SC, Luoma JB, Bond FW, Masuda A, Lillis J. Acceptance and commitment therapy: model, processes and outcomes. Behaviour Research and Therapy 2006; 44(1):1-25. [DOI: 10.1016/j.brat.2005.06.006] [PMID: 16300724]

\section{Herman 1992}

Herman JL. Complex PTSD: a syndrome in survivors of prolonged and repeated trauma. Journal of Traumatic Stress 1992; 5(3):377-91. [DOI: 10.1002/jts.2490050305]

\section{Home Office 2017}

Home Office. The role of the Independent Sexual Violence Adviser: essential elements. bit.ly/2En4iOv (accessed 3 January 2019).

\section{Houghton 2017}

Houghton C, Dowling M, Meskell P, Hunter A, Gardner H, Conway $\mathrm{A}$, et al. Factors that impact on recruitment to randomised trials in health care: a qualitative evidence synthesis. Cochrane Database of Systematic Reviews 2017, Issue 5. [DOI: 10.1002/14651858.MR000045]

\section{IOM 2015}

Institute of Medicine. Psychosocial Interventions for Mental and Substance Use Disorders: A Framework for Establishing Evidence-Based Standards. Washington (DC): National Academies Press, 2015

\section{Jewkes 2002}

Jewkes R, Sen P, Garcia-Moreno C. Sexual violence. In: Krug EG, Dahlberg LL, Mercy JA, Zwi AB, Lozano R, editor(s), editors(s). World Report on Violence and Health. Geneva $(\mathrm{CH})$ : World Health Organization, 2002:147-81.

\section{Jewkes 2011}

Jewkes R, Sikweyiya Y, Morrell R, Dunkle K. Gender inequitable masculinity and sexual entitlement in rape perpetration South Africa: findings of a cross-sectional study. PLOS One 2011; 6(12):e29590. [DOI: 10.1371/journal.pone.0029590] [PMC3247272] [PMID: 22216324]

\section{Kelly 1966}

Kelly JG. Ecological constraints on mental health services. American Psychologist 1966; 21(6):535-9. [DOI: 10.1037/ h0023598]

\section{Kelly 1968}

Kelly JG. Towards an ecological conception of preventive interventions. In: Carter J, editors(s). Research Contributions from Psychology to Community Mental Health. New York (NY): Behavioral Publications, 1968:75-99. [files.eric.ed.gov/fulltext/ ED022220.pdf]

\section{Kelly 1971}

Kelly JG. Qualities for the community psychologist. American Psychologist 1971; 26(10):897-903. [DOI: 10.1037/h0032231]

\section{Kilpatrick 1983}

Kilpatrick DG, Veronen LJ. Treatment for rape-related problems: crisis intervention is not enough. In: Cohen LH, Claiborn WL, Spector CA, editors(s). Crisis Intervention. New York (NY): Human Sciences Press, 1983:165-85.

\section{Koss 1987}

Koss MP, Harvey MR. The Rape Victim: Clinical and Community Approaches to Treatment. Lexington (MA): Stephen Greene Press, 1987.

\section{Koss 1991}

Koss MP, Harvey MR. The Rape Victim: Clinical and Community Interventions. 2 edition. Newbury Park (CA): Sage Publications, Inc, 1991.

\section{Krug 2002}

Krug EG, Dahlberg LL, Mercy JA, Zwi AB, Lozano R, . World Report on Violence and Health. Geneva $(\mathrm{CH})$ : World Health Organization, 2002.

\section{Leach 2016}

Leach C, Stuart A, Smallbone S. Testing the sexually abusedsexual abuser hypothesis: a prospective longitudinal birth cohort study. Child Abuse \& Neglect 2016; 51:144-53. [DOI: 10.1016/j.chiabu.2015.10.024] [PMID: 26615777]

\section{Lerman 2019}

Lerman I, Davis B, Huang M, Huang C, Sorkin L, Proudfoot J, et al. Noninvasive vagus nerve stimulation alters neural response and physiological autonomic tone to noxious thermal

Survivor, family and professional experiences of psychosocial interventions for sexual abuse and violence: a qualitative evidence 
challenge. PLOS ONE 2019; 14(2):e0201212. [DOI: 10.1371/ journal.pone.0201212] [PMC6373934] [PMID: 30759089]

\section{Letourneau 2018}

Letourneau EJ, Brown DS, Fang X, Hassan A, Mercy JA. The economic burden of child sexual abuse in the United States. Child Abuse \& Neglect 2018; 79:413-22. [DOI: 10.1016/ j.chiabu.2018.02.020] [PMC6542279] [PMID: 29533869]

\section{Lewin 2018}

Lewin S, Booth A, Glenton C, Munthe-Kaas H, Rashidian A, Wainwright $M$, et al. Applying GRADE-CERQual to qualitative evidence synthesis findings: introduction to the series. Implementation science 2018; 13(Suppl 1):2. [DOI: 10.1186/ s13012-017-0688-3] [PMC5791040] [PMID: 29384079]

\section{London 2008}

London K, Bruck C, Wright DB, Ceci SJ. Review of the contemporary literature on how children report sexual abuse to others: findings, methodological issues, and implications for forensic interviewers. Memory 2008; 16(1):29-47. [DOI: 10.1080/09658210701725732] [PMID: 18158687]

\section{Macdonald 2012}

Macdonald G, Higgins JPT, Ramchandani P, Valentine JC, Bronger LP, Klein P, et al. Cognitive-behavioural interventions for children who have been sexually abused. Cochrane Database of Systematic Reviews 2012, Issue 5. [DOI: 10.1002/14651858.CD001930.pub3] [PMID: 22592679]

\section{McKibbon 2006}

McKibbon KA, Wilczynski NL, Haynes RB. Developing optimal search strategies for retrieving qualitative studies in PsycINFO. Evaluation \& the Health Professions 2006; 29(4):440-54. [DOI: 10.1177/0163278706293400] [PMID: 17102065]

\section{Miller 2015}

Miller KE, Cranston CC, Davis JL, Newman E, Resnick H. Psychological outcomes after a sexual assault video intervention: a randomized trial. Journal of Forensic Nursing 2015; 11(3):129-36. [DOI: 10.1097/JFN.0000000000000080] [PMID: 26291847]

\section{Moher 2009}

Moher D, Liberati A, Tetzlaff J, Altman DG, PRISMA Group. Preferred reporting items for systematic reviews and meta-analyses: the PRISMA statement. Annals of Internal Medicine 2009; 151(4):264-9. [DOI: 10.7326/0003-4819-151-4-200908180-00135] [PMID: 19622511]

\section{Moore 2015}

Moore GF, Audrey S, Barker M, Bond L, Bonell C, Hardeman W, et al. Process evaluation of complex interventions: Medical Research Council guidance. BMJ 2015; 350:h1258. [DOI: 10.1136/bmj.h1258] [PMC4366184] [PMID: 25791983]

\section{Morgan 2017}

Morgan RE, Kena G. Criminal Victimization, 2016. Washington (DC): Bureau of Justice Statistics, 2017.

\section{Neville 1999}

Neville HA, Heppner MJ. Contextualizing rape: reviewing sequelae and proposing a culturally inclusive ecological model of sexual assault recovery. Applied \& Preventive Psychology 1999; 8(1):41-62. [DOI: 10.1016/S0962-1849(99)80010-9]

\section{NHS 2015}

NHS England. Commissioning Framework for Adult and Paediatric Sexual Assault Referral Centres (SARC) Services. London (UK): NHS England, 2015.

\section{Noyes 2018}

Noyes J, Booth A, Lewin S, Carlsen B, Glenton C, Colvin CJ, et al. Applying GRADE-CERQual to qualitative evidence synthesis findings - paper 6: how to assess relevance of the data. Implementation Science 2018; 13(Suppl 1):4. [DOI: 10.1186/ s13012-017-0693-6] [PMC5791042] [PMID: 29384080]

\section{Noyes 2019}

Noyes J, Booth A, Cargo M, Flemming K, Harden A, Harris J, et al. Chapter 21: Qualitative evidence. In: Higgins JPT, Thomas J, Chandler J, Cumpston M, Li T, Page MJ, Welch VA (editors). Cochrane Handbook for Systematic Reviews of Interventions version 6.0 (updated July 2019). Cochrane, 2019. Available from www.training.cochrane.org/handbook.

\section{O'Doherty 2016}

O'Doherty L, Taket A, Valpied J, Hegarty K. Receiving care for intimate partner violence in primary care: barriers and enablers for women participating in the weave randomised controlled trial. Social Science \& Medicine 2016; 160:35-42. [DOI: 10.1016/ j.socscimed.2016.05.017] [PMID: 27208669]

\section{ONS 2015}

Office for National Statistics. Violent crime and sexual offences - intimate personal violence and serious sexual assault. In: Findings from the 2013/14 Crime Survey for England and Wales and Police Recorded Crime Over the Same Period on Violent Crime and Sexual Offences. London (UK): Office for National Statistics, 2015.

\section{Pereda 2009}

Pereda N, Guilera G, Forns M, Gómez-Benito J. The prevalence of child sexual abuse in community and student samples: a meta-analysis. Clinical Psychology Review 2009; 29(4):328-38. [DOI: 10.1016/j.cpr.2009.02.007] [PMID: 19371992]

\section{Peterson 2017}

Peterson C, DeGue S, Florence C, Lokey CN. Lifetime economic burden of rape among US adults. American Journal of Preventive Medicine 2017; 52(6):691-701. [DOI: 10.1016/ j.amepre.2016.11.014] [PMC5438753] [PMID: 28153649]

\section{Pietrzak 2011}

Pietrzak RH, Goldstein RB, Southwick SM, Grant BF. Prevalence and Axis I comorbidity of full and partial posttraumatic stress disorder in the United States: results from wave 2 of the National Epidemiologic Survey on Alcohol and Related Conditions. Journal of Anxiety Disorders 2011; 25(3):456-65. [DOI: 10.1016/j.janxdis.2010.11.010] [PMC3051041] [PMID: 21168991] 


\section{Pitman 1996}

Pitman RK, Orr SP, Altman B, Longpre RE, Poiré RE, Macklin ML. Emotional processing during eye movement desensitization and reprocessing therapy of Vietnam veterans with chronic posttraumatic stress disorder. Comprehensive Psychiatry 1996; 37(6):419-29. [DOI: 10.1016/s0010-440x(96)90025-5] [PMID: 8932966]

\section{Resick 1988}

Resick PA, Jordan CG, Girelli SA, Hutter CK, Marhoefer-Dvorak S. A comparative outcome study of behavioral group therapy for sexual assault victims. Behavior Therapy 1988; 19(3):385-401. [DOI: 10.1016/S0005-7894(88)80011-X]

\section{Resnick 1997}

Resnick HS, Yehuda R, Acierno R. Acute post-rape plasma cortisol, alcohol use, and PTSD symptom profile among recent rape victims. Annals of the New York Academy of Sciences 1997; 821:433-6. [DOI: 10.1111/j.1749-6632.1997.tb48298.x] [PMID: 9238223]

\section{Review Manager 2014 [Computer program]}

Nordic Cochrane Centre, The Cochrane Collaboration Review Manager 5 (RevMan 5). Version 5.3. Copenhagen: Nordic Cochrane Centre, The Cochrane Collaboration, 2014.

\section{Ring 2011}

Ring NA, Ritchie K, Mandava L, Jepson R. A Guide to Synthesising Qualitative Research for Researchers Undertaking Health Technology Assessments and Systematic Reviews. Edinburgh (UK): NHS Quality Improvement Scotland, 2011.

\section{Rothbaum 1997}

Rothbaum BO. A controlled study of eye movement desensitization and reprocessing in the treatment of posttraumatic stress disordered sexual assault victims. Bulletin of the Menninger Clinic 1997; 61(3):317-34. [PMID: 9260344]

\section{Saied-Tessier 2014}

Saied-Tessier A. Estimating the Costs of Child Sexual Abuse in the UK. London (UK): NSPCC, 2014.

\section{Sandelowski 2002}

Sandelowski M, Barroso J. Finding the findings in qualitative studies. Journal of Nursing Scholarship 2002; 34(3):213-9. [DOI: 10.1111/j.1547-5069.2002.00213.x] [PMID: 12237982]

\section{Shapiro 1995}

Shapiro F. Eye Movement Desensitization and Reprocessing: Basic Principles, Protocols, and Procedures. New York (NY): Guilford Press, 1995.

\section{Sikkema 2018}

Sikkema KJ, Choi KW, Robertson C, Knettel BA, Ciya N, Knippler ET, et al. Development of a coping intervention to improve traumatic stress and HIV care engagement among South African women with sexual trauma histories. Evaluation and Program Planning 2018; 68:148-56. [DOI: org/10.1016/ j.evalprogplan.2018.02.007] [PMC5953816] [PMID: 29597104]

\section{Singer 2003}

Singer M, Clair S. Syndemics and public health: reconceptualizing disease in bio-social context. Medical Anthropology Quarterly 2003; 17(4):423-41. [DOI: 10.1525/ maq.2003.17.4.423] [PMID: 14716917]

\section{Suri 2011}

Suri H. Purposeful sampling in qualitative research synthesis. Qualitative Research Journal 2011; 11(2):63-75. [DOI: 10.3316/ QRJ1102063]

\section{Tewkesbury 2007}

Tewkesbury R. Effects of sexual assaults on men: physical, mental and sexual consequences. International Journal of Men's Health 2007; 6(1):22-35. [DOI: 10.3149/jmh.0601.22]

\section{Thomas 2008}

Thomas J, Harden A. Methods for the thematic synthesis of qualitative research in systematic reviews. $B M C$ Medical Research Methodology 2008; 8:45. [DOI: 10.1186/ 1471-2288-8-45] [PMC2478656] [PMID: 18616818]

\section{Thomas 2010 [Computer program]}

Social Science Research Unit, UCL Institute of Education. EPPIReviewer 4.0: software for research synthesis. EPPI-Centre software. Thomas J, Brunton J, Graziosi S. London (UK): Social Science Research Unit, UCL Institute of Education., 2010. [DOI: 10.1186/1471-2288-8-45]

\section{Turner 2016}

Turner HA, Shattuck A, Finkelhor D, Hamby S. Polyvictimization and youth violence exposure across contexts. Journal of Adolescent Health 2016; 58(2):208-14. [DOI: 10.1016/ j.jadohealth.2015.09.021] [PMID: 26598060]

\section{Vandenberghe 2018}

Vandenberghe A, Hendriks B, Peeters L, Roelens K, Keygnaert I. Establishing Sexual Assault Care Centres in Belgium: health professionals' role in the patient-centred care for victims of sexual violence. BMC Health Services Research 2018; 18(1):807. [DOI: 10.1186/s12913-018-3608-6] [PMC6196455] [PMID: 30348151]

\section{Veronen 1983}

Veronen LJ, Kilpatrick DG. Stress management for rape victims. In: Meichenbaum D, Jaremko ME, editor(s), editors(s). Stress Reduction and Prevention. New York (NY): Plenum Press, 1983:341-74.

\section{Vickerman 2009}

Vickerman KA, Margolin G. Rape treatment outcome research: empirical findings and state of the literature. Clinical Psychology Review 2009; 29(5):431-48. [DOI: 10.1016/j.cpr.2009.04.004] [PMC2773678] [PMID: 19442425]

\section{Walters 2006}

Walters LA, Wilczynski NL, Haynes RB, Hedges Team. Developing optimal search strategies for retrieving clinically relevant qualitative studies in EMBASE. Qualitative Health Research 2006; 16(1):162-8. [DOI: 10.1177/1049732305284027] [PMID: 16317183] 


\section{Waters 2013}

Walters ML, Chen J, , . The National Intimate Partner and Sexual Violence Survey (NISVS): 2010 Findings on Victimization by Sexual Orientation. Atlanta (GA): National Center for Injury Prevention and Control; Centers for Disease Control and Prevention, 2013.

\section{WHO 2005}

World Health Organization. World Health Organisation multicountry study on women's health and domestic violence against women. Initial results on prevalence, health outcomes and women's responses. World Health Organization 2005.

\section{WHO 2013}

World Health Organisation. Global and Regional Estimates of Violence Against Women: Prevalence and Health Effects of Intimate Partner Violence and Non-Partner Sexual Violence. Geneva (CH): World Health Organisation, 2013.

\section{WHO/PAHO 2012}

World Health Organization, Pan American Health Organization. Understanding and Addressing Violence Against Women: Human Trafficking. Geneva $(\mathrm{CH})$ : World Health Organization, 2012.

\section{AP PE N DICES}

\section{Appendix 1. MEDLINE search strategy}

Lines 78-81 use search terms from the best optimization version of Wong's search strategy to detect qualitative studies in MEDLINE (Wong 2004), with the addition of the MeSH term Qualitative research/, an indexing term introduced after publication of this filter.

1 sex offenses/

2 Child Abuse, Sexual/

3 "Adult Survivors of Child Abuse"/

4 Incest/

5 Intimate partner violence/

6 human trafficking/

7 rape/

8 Spouse abuse/

9 (rape or raped or incest\$).tw,kf.

10 (sex\$ adj3 (abuse or assaul\$ or attack\$ or aggress\$ or coer\$ or exploit\$ or force or molest $\$$ or offen\$ or traffick\$ or trauma\$ or unlawful $\$$ or unwanted or violen\$)).tw,kf.

11 (intercourse adj3 (coer\$ or force\$ or unwanted)).tw,kf.

12 (sex\$ adj1 (victim\$ or revictim\$ or re-victim\$ or survivor\$)).tw,kf.

13 intimate partner violence.tw,kf.

14 or/1-13

15 Adaptation, Psychological/

16 exp Behavior Therapy/

17 Combined Modality Therapy/

18 community networks/

19 exp Complementary therapies/

20 exp Counseling/

21 Exercise/

22 Exercise therapy/

23 Health Education/

24 Health Knowledge, Attitudes, Practice/

25 Interview, Psychological/

26 exp mind body therapies/

27 Psychological adjustment/

28 psychosocial support systems/

\section{Wilczynski 2007}

Wilczynski NL, Marks S, Haynes RB. Search strategies for identifying qualitative studies in CINAHL. Qualitative Health Research 2007; 17(5):705-10. [DOI: 10.1177/1049732306294515] [PMID: 17478652]

\section{Willen 2017}

Willen SS, Knipper M, Abadía-Barrero CE, Davidovitch N. Syndemic vulnerability and the right to health. Lancet 2017; 389(10072):964-77. [DOI: 10.1016/S0140-6736(17)30261-1] [PMID: 28271847]

\section{Wirtz 2018}

Wirtz AL, Poteat TC, Malik M, Glass N. Gender-based violence against transgender people in the United States: a call for research and programming. Trauma, Violence \& Abuse 2018 Feb 13 [Epub ahead of print]. [DOI: 10.1177/1524838018757749] [PMID: 29439615 ]

\section{Wong 2004}

Wong SS, Wilczynski NL, Haynes RB, Hedges Team. Developing optimal search strategies for detecting clinically relevant qualitative studies in MEDLINE. Studies in Health Technology and Informatics 2004; 107(Pt 1):311-6. [DOI: 10.3233/978-1-60750-949-3-311] [PMID: 15360825]

Survivor, family and professional experiences of psychosocial interventions for sexual abuse and violence: a qualitative evidence 
29 exp psychotherapy/

30 "Referral and Consultation"/

31 Self-Help Groups/

32 Social Support/

33 video recording/ or videotape recording/

34 Writing/

35 ((abreaction or desensitization or exposure or implosive) adj3 therap\$).tw,kf.

36 "acceptance and commitment therapy".tw,kf.

37 (advisor\$ or advo78-91 arecate\$ or advocacy).tw,kf.

38 ( (animal\$ or art or colo?r or creative\$ or dance or dancing or drama or equine or experiential or music or narrative or play\$ or sensory or singing) adj3 (program\$ or intervention\$ or therap\$)).tw,kf.

39 (autogenic or autosuggestion $\$$ or auto-suggestion $\$$ or breathing exercise $\$$ or hypnosis or hypno-therapy or hypnotherapy).tw,kf.

40 behavio $\$$ activation.tw,kf.

41 (behavio?r\$ adj3 (intervention\$ or program\$ or therap\$ or training or treatment\$)).tw,kf.

42 ((biofeedback or feedback or imagery) adj3 (intervention $\$$ or therap\$ or train $\$$ or treatment\$)).tw, kf.

43 ((brief or combination or compass\$ focus\$ or integrated or integrative or time-limited) adj3 (intervention\$ or therap\$ or treatment

\$)).tw,kf.

44 ((client focus\$ or non-direct\$ or nondirect\$ or solution focus\$ or trauma\$ or talking) adj3 therap\$).tw,kf.

45 (cognitiv\$ or cognition).tw,kf.

46 CBT.tw, kf.

47 ((cope or coping) adj1 (intervention\$ or mechanism\$ or skill\$ or technique\$)).tw,kf.

48 counsel?ing.tw,kf.

49 ((couple\$ or family or group or systemic\$ or multimodal\$ or multi-modal\$) adj3 (program\$ or intervention\$ or therap\$ or treat\$)).tw, kf.

50 dialectical behavio? $\mathbf{s}$ therap\$.tw,kf.

51 (exercise $\$$ or physical training).tw,kf.

52 ((existential or gestalt or humanistic or interpersonal or milieu or person-centred or residential or socioenvironmental or socioenvironmental) adj therap\$).tw,kf.

53 expressive writing.tw,kf.

54 ("Eye Movement Desensitization and Reprocessing" or EMDR).tw,kf.

55 (meditat\$ or mental training or mindfulness\$ or mind training or brain training or yoga).tw, kf.

56 motivational interview\$.tw,kf. (3315)

57 (reality therap\$ or problem solving).tw,kf.

58 (psycho\$ therap\$ or psychotherap\$).tw,kf.

59 (psychoanalytic\$ or psycho-analytic\$ or psychodynamic\$ or psycho-dynamic\$).tw,kf.

60 (psychodrama or psycho-drama or acting out or role play).tw,kf. (2558)

61 (psychosocial or psycho-social or psychoeducation\$ or psycho-education\$).tw,kf.

62 rational emotive.tw,kf.

63 (Relax\$ adj3 (training\$ or treatment\$ or therap\$)).tw,kf.

64 (Service\$ adj3 (refer\$ or use\$)).tw,kf.

65 (stress inoculation training or SIT or prolonged exposure therapy or PET or cognitive processing therapy or CPT).tw, kf.

66 ((support or advice or advis\$1) adj1 (centre $\$ 1$ or center $\$ 1$ or community or group $\$$ or network $\$$ or social or staff\$)).tw,kf.

67 (therapeutic allianc\$ or therapeutic relationship\$ or therapeutic communit\$).tw,kf.

68 Third wave.tw,kf.

69 (th or rh).fs.

70 (care or caring or heal or healing or intervention\$ or recover\$ or rehabilit\$ or support\$ or therap\$ or treat\$).ti,kf.

71 or/15-70

7214 and 71

73 (rape adj3 (centre\$ or center\$ or service\$ or support)).tw,kf.

74 ((sex\$ assault adj3 centre\$) or (sex\$ assault adj3 center\$) or (sex\$ assault adj3 service\$) or (sex\$ assault adj3 support)).tw,kf.

75 ((sex\$ abuse $\$$ adj3 centre\$) or (sex\$ abuse $\$$ adj3 center $\$$ ) or (sex $\$$ abuse $\$$ adj3 service\$) or (sex $\$$ abuse $\$$ adj3 support)).tw,kf.

76 or $/ 73-75$

7772 or 76

78 Interview/ or interview\$.mp.

79 Qualitative research/ or qualitative.tw,kf.

80 experience $\$ . m p$.

81 or $/ 78-80$

8277 and 81

\section{H I S T O R Y}

Protocol first published: Issue 6, 2020

Survivor, family and professional experiences of psychosocial interventions for sexual abuse and violence: a qualitative evidence synthesis (Protocol)

Copyright (c) 2020 The Cochrane Collaboration. Published by John Wiley \& Sons, Ltd. 


\section{CONTRIBUTIONS OF AUTHORS}

SB and LOD developed the concept for the review. SB, LOD, NK and GC drafted the protocol. KB, RC, GF, and EH reviewed the draft. With input from LOD, SB led the integration of the comments and feedback from the co-authors and the reviewers, and submission of the final version. SB is the guarantor for the review.

\section{DECLARATIONS OF INTEREST}

With the exception of EH, all review authors are funded for their work on this review by the Multidisciplinary Evaluation of Sexual Assault Referral Centres for better Health (MESARCH) project; a project (number 16/117/04) funded by an institutional research grant from the National Institute for Health Research (NIHR) Health Service and Delivery Research (HS\&DR) Programme to Coventry University. The views expressed are those of the authors and not necessarily those of the NHS, NIHR or Department of Health.

SB declares no other conflicts of interest.

NK declares no other conflicts of interest.

GC declares no other conflicts of interest.

KB declares no other conflicts of interest.

$\mathrm{RC}$ declares no other conflicts of interest. In the interests of transparency, RC also declares that she works as a medical doctor and has been funded through her workplace (NHS) to carry out a PhD in a related area - disclosure of sexual violence in sexual health settings.

EH declares no other conflicts of interest.

GF declares that he is Chief Investigator or Co-Investigator on a range of NIHR and MRC (Medical Research Council) grants, including an NIHR HS\&RD grant from which this review is funded.

LOD declares that she is Chief Investigator on the MESARCH project.

\section{SOURCES OF SUPPORT}

\section{Internal sources}

- Coventry University, UK

$20 \%$ of the MESARCH (Multidisciplinary Evaluation of Sexual Assault Referral Centres for better Health) project is funded by Coventry University.

\section{External sources}

- National Institute for Health Research (NIHR) Health Services and Delivery Research (HS\&DR) Programme, UK $80 \%$ of the MESARCH project is funded by the NIHR HS\&DR Programme. 